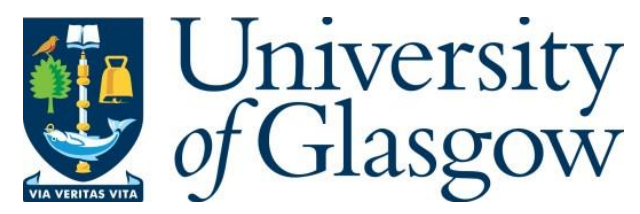

Wang, Z., Zhou, K., Li, S. and Yang, Y. (2018) Fractional-Order Time Delay

Compensation in Deadbeat Control for Power Converters. In: 2018 IEEE International Power Electronics and Application Conference and Exposition (PEAC), Shenzhen, China, 04-07 Nov 2018, ISBN 9781538660546.

There may be differences between this version and the published version. You are advised to consult the publisher's version if you wish to cite from it.

http://eprints.gla.ac.uk/192825/

Deposited on: 13 August 2019

Enlighten - Research publications by members of the University of Glasgow http://eprints.gla.ac.uk 


\section{Fractional-order time delay compensation in deadbeat control for power converters}

\author{
Zuo Wang \\ School of Automation \\ Southeast University \\ Nanjing, 210096, P.R. China \\ z.wang@seu.edu.cn
}

\author{
Keliang Zhou \\ School of Engineering \\ University of Glasgow \\ Glasgow, G12 QQ, United Kingdom \\ keliang.zhou@glasgow.ac.uk \\ Yongheng Yang \\ Department of Energy Technology \\ Aalborg University \\ Aalborg, Denmark \\ yoy@et.aau.dk
}

\author{
Shihua Li \\ School of Automation \\ Southeast University \\ Nanjing, 210096, P.R. China \\ 1 sh@seu.edu.cn
}

\begin{abstract}
Deadbeat control scheme is widely implemented in the control of power electronics and electrical drives, which is of simplification, rapidity and flexibility. However, owing to its sensitive to model uncertainties and unmodeled dynamics, the practical control performance is severely degraded and sometimes even unstable. Uncertain time delay is a typical case of model uncertainties, which severely deteriorates the control accuracy and dramatically reduce the system stability margin of deadbeat control. In this paper, the time delay effects on the control performance and system stability are investigated. A fractional-order Smith Predictor based solution is proposed to compensate arbitrary time delay with high accuracy, simple structure, and good robustness. The composite control scheme offers accurate time delay compensations in digital implementation and considerably enhances the robustness of the control system, which will effectively promote widespread applications of the deadbeat scheme. An application example of three-phase inverter system is explored to comprehensively illustrate the feasibility and effectiveness of the proposed scheme.
\end{abstract}

Keywords-Deadbeat control, time delay compensation, fractional-order, Smith Predictor, power converters.

\section{INTRODUCTION}

Deadbeat control scheme has aroused a great deal of interests in the control of power electronics and electrical drives, which is of simplification, rapidity and flexibility [1]-[8]. As compared with other approaches, it is able to bring zero steady-state tracking errors within minimum number of steps[3]. However, deadbeat approaches are sensitive to model uncertainties and unmodeled dynamics [4]-[8]. Their practical control performance confronts severe degradation problem in the presence of model uncertainties. Therefore, model uncertainties are regarded as challenge issues, which limit widespread applications of deadbeat control in practice.

Among all the factors, the uncertain time delay is considered as a critical one [3], [9], [10]. Many reasons lead to the time delay problem in power electronics, such as the time consumed in measurement convention, control law

This work was partially supported by National Natural Science Foundation of China under Grants (61673305), and Scientific Research Foundation of Graduate School of Southeast University (YBJJ1766). calculation and actuation, as well as unmodeled nonlinearity of the devices. The time delay effects in the closed-loop characteristic equation will result in serious deterioration in control accuracy and dramatic reduction of system stability margin [11]-[13].

The problem of time delay compensation in deadbeat control for power converters are considered but not solved systematically, by far. A linear phase-lead compensation solution is successfully employed in repetitive control systems to compensate the time delay [14]-[16]. However, it is impractical to be adopted in the conventional deadbeat control frame due to its incausal lead-time item. A state estimator is adopted for compensation of computational delay [3]. Also focused on this problem, another simple design method of two steps forward prediction approach is proposed in the frame of model predictive control [10], [17], [18]. In these solutions, computational delay effects are effectively removed and control accuracy is prominently improved. However, as mentioned above, apart from computational delay, many other factors lead to the delay problem in practical systems. In these cases, the above mentioned approaches are not suitable and fail to achieve satisfying control performance. Therefore, a universal delay compensation approach for the deadbeat control schemes should be investigated in practical applications.

The Smith Predictor is known as an effective and powerful delay compensation approach and has been applied in various control systems, e.g., power electronic systems [4], [19], remote control systems [20], and process control systems [21]. It provides insight into how universal delay compensation problem can be effectively solved. However, the conventional integer-order Smith Predictor confronts severe frequency robustness problems in practice. It is sensitive to the ratio of the sampling frequency to the delay frequency. Integer number of delay units $\left(z^{-N}\right)$ are adopted in digital Smith Predictor, where the delay unit number $N=T_{d} / T_{s}$ with $T_{s}$ being the sampling period and $T_{d}$ being the time delay value. The integer number of delay units with a given sampling rate is not able to accurately compensate a fractional time delay, and degrades the compensation performance, especially in the low sampling frequency cases [15], [22]. It is inflexible to change the system sampling rate in the real-time power converter 
systems to achieve a better time delay compensation performance [22]-[25].

Addressing it, a fractional-order Smith Predictor based universal delay compensation approach for deadbeat control is proposed in this paper. In the presented scheme, arbitrary time delay values are approximated accurately by fractionalorder Smith Predictor, which shows a nice frequency robustness. By combining with the universal delay compensation, it is able to achieve high control accuracy and superior robustness performance, which effectively promotes widespread applications of the deadbeat control schemes in practical systems. Comprehensive analysis and synthesis method for the proposed control system are given. Simulation verifications of the proposed control scheme on three-phase inverter systems are carried out to demonstrate the feasibility and effectiveness of the proposed scheme under various time delay scenarios.

\section{UNIVERSAL COMPENSATOR DESIGN FOR ARBITRARY TIME DEALY}

\section{A. Unifie Conventional Smith Predictor Based Solution}

In the presence of time delay, the conventional deadbeat control approaches fail to achieve satisfying control performance. Smith Predictor has been proved to be an effective delay compensation solution. The implementation of conventional Smith Predictor based control approach is illustrated in Fig. 1, where $G_{c}(z)$ is the designed controller, $G_{p}(z) z^{-N}$ is the actual system model with time delay

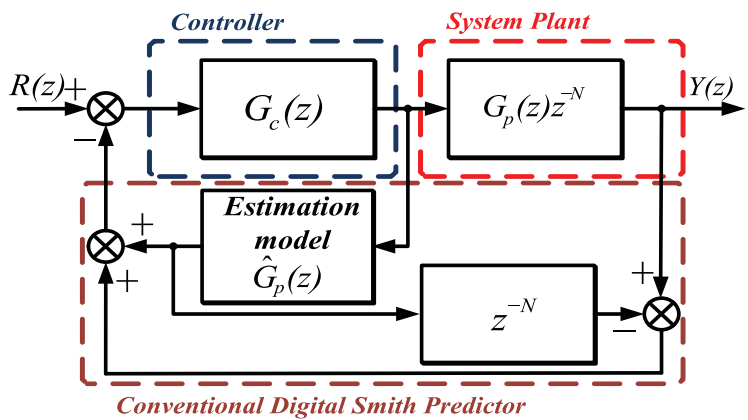

Fig. 1. Implementation of conventional Smith Predictor based control approach.

and $G_{d}(z)=\hat{G}_{p}(z)\left(1-z^{-N}\right)$ is the time delay compensation based on the Smith Predictor.

Then the pulse transfer function of closed-loop system can be expressed as follows:

$$
\frac{Y(z)}{R(z)}=\frac{G_{c}(z) G_{p}(z) z^{-N}}{1+G_{c}(z) G_{d}(z)+G_{c}(z) G_{p}(z) z^{-N}} .
$$

It is not so difficult to obtain the accurate model of the system, which means $\hat{G}_{p}(z) \approx G_{p}(z)$. In this case, the pulse transfer function of closed-loop system can be reduced to:

$$
\frac{Y(z)}{R(z)} \approx \frac{G_{c}(z) G_{p}(z)}{1+G_{c}(z) G_{p}(z)} z^{-N} .
$$

With the application of Smith Predictor, it is easy to observe that the system stability is guaranteed by separating time delay part from the nominal system. Time delay effects are removed from the closed-loop characteristic equation, which improves the system stability margin without

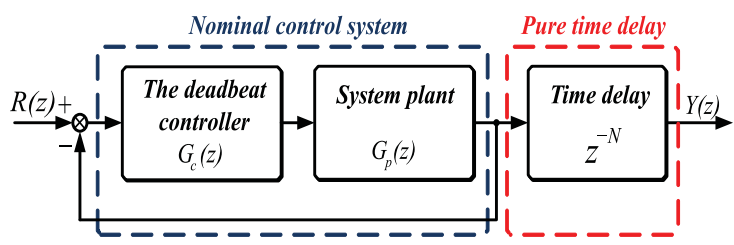

Fig. 2. The equivalent control system of the delay compensation based deadbeat control.

sacrificing the dynamic performance. Therefore, the block diagram in Fig. 1 can be simplified into the nominal control system with a pure time delay term, as shown in Fig. 2

Despite the benefits mentioned above, there is a main frequency robustness problem of conventional integer-order Smith Predictor in digital implementation, which constrains the delay compensation performance in practical power electronics.

\section{B. Fractional-Order Smith Predictor}

When facing mismatches between time delay $T_{d}$ and sampling rate $1 / T_{s}$, there will always confront severe frequency robustness problem. In this case, the integer number of delay units with a given sampling rate is not able to accurately compensate a fractional time delay and degrades the performance, especially in the low sampling frequency cases.

In the fractional time delay case, assuming that the delay units can be expressed as $z^{-N}=z^{-N_{i}-F}$ with $N_{i}=$ int $[N]$ being the integer part of $N$ and $F$ being the fractional part. The fractional time delay part $z^{-F}$ is impractical to be realized directly in digital controllers. Instead, in this paper the fractional part is approximated and constructed by fractional delay filters with integer orders according to the Lagrange interpolation polynomial finite-impulse-response (FIR) filter design method [22]-[25].

The fractional delay part $z^{-F}$ can be constructed as follows:

$$
z^{-F} \approx \sum_{k=0}^{p} A_{k} z^{-k}
$$

where $k=0,1, \ldots, p$ and the coefficient $A_{k}$ can be obtained as:

$$
A_{k}=\prod_{i=0, i \neq k}^{p} \frac{F-i}{k-i},(k, i=0,1, \ldots, p) .
$$

In order to demonstrate the approximation accuracy of the fractional delay $z^{-F}$, the magnitude responses of the proposed scheme with order $p=1$ and $p=2$ for various $F$ values are illustrated in Fig. 3. It is seen that the constructed fractional delay part is of high approximation accuracy at low frequency within the bandwidth of $50 \%$ of the Nyquist frequency for the filter of order $p=1$ and within the bandwidth of $61 \%$ for order $p=2$. Within this pass band, arbitrary time delay can be accurately compensated by the fractional-order Smith Predictor, which shows a nice frequency robustness. Specifically, in practical applications, for keeping a balance between simplify and approximation accuracy, the degree $p$ is usually chosen as $p=2$. In this case, a linear interpolation polynomial 


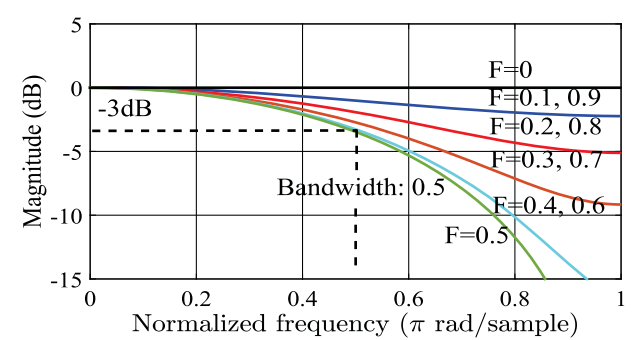

(a)

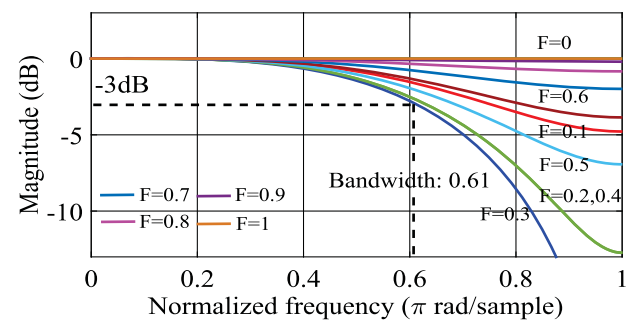

(b)

Fig. 3. Magnitude responses of the fractional-order filters. (a) $p=1$. (b)

$$
p=2 \text {. }
$$

$z^{-F} \approx \frac{(F-1)(F-2)}{2}-F(F-2) z^{-1}+\frac{F(F-1)}{2} z^{-2}$ can be obtained.

Remark 1: In the absence of time delay, it is derived from (4) that $A_{0}=1, A_{k}=0,(k=1,2, \ldots, p)$, which results in the time delay compensation part $G_{d}(z) \equiv 0$. It implies that the proposed universal fractional-order Smith Predictor works as a patch to the controller and the performance would be no worse than the standard deadbeat current control scheme.

\section{CASe Study of A Three-Phase INVERTER System}

\section{A. Modeling Three-Phase PWM Inverter}

The electrical dynamics model of a three-phase PWM inverter with resistor loads in Fig. 4 can be described as follows:

$$
\begin{aligned}
& {\left[\begin{array}{llllll}
1 & 0 & -1 & 0 & 0 & 0 \\
-1 & 1 & 0 & 0 & 0 & 0 \\
0 & -1 & 1 & 0 & 0 & 0 \\
0 & 0 & 0 & 1 & -1 & 0 \\
0 & 0 & 0 & 0 & 1 & -1 \\
0 & 0 & 0 & -1 & 0 & 1
\end{array}\right]\left[\begin{array}{l}
\dot{v}_{a b} \\
\dot{v}_{b c} \\
\dot{v}_{c a} \\
\dot{i}_{a} \\
\dot{i}_{b} \\
\dot{i}_{c}
\end{array}\right]=\left[\begin{array}{llllll}
\frac{-1}{R C} & 0 & \frac{1}{R C} & \frac{1}{C} & 0 & 0 \\
\frac{1}{R C} & \frac{-1}{R C} & 0 & 0 & \frac{1}{C} & 0 \\
0 & \frac{1}{R C} & \frac{-1}{R C} & 0 & 0 & \frac{1}{C} \\
\frac{-1}{L} & 0 & 0 & 0 & 0 & 0 \\
0 & \frac{-1}{L} & 0 & 0 & 0 & 0 \\
0 & 0 & \frac{-1}{L} & 0 & 0 & 0
\end{array}\right]\left[\begin{array}{l}
v_{a b} \\
v_{b c} \\
v_{c a} \\
i_{a} \\
i_{b} \\
i_{c}
\end{array}\right]} \\
& +\left[\begin{array}{ccc}
0 & 0 & 0 \\
0 & 0 & 0 \\
0 & 0 & 0 \\
\frac{1}{L} & 0 & 0 \\
0 & \frac{1}{L} & 0 \\
0 & 0 & \frac{1}{L}
\end{array}\right]\left[\begin{array}{l}
v_{A B} \\
v_{B C} \\
v_{C A}
\end{array}\right]
\end{aligned}
$$

(5)

where the output line-to-line voltages $v_{a b}, v_{b c}$, and $v_{c a}$; and the phase currents $i_{a}, i_{b}$, and $i_{c}$ are the state variables;

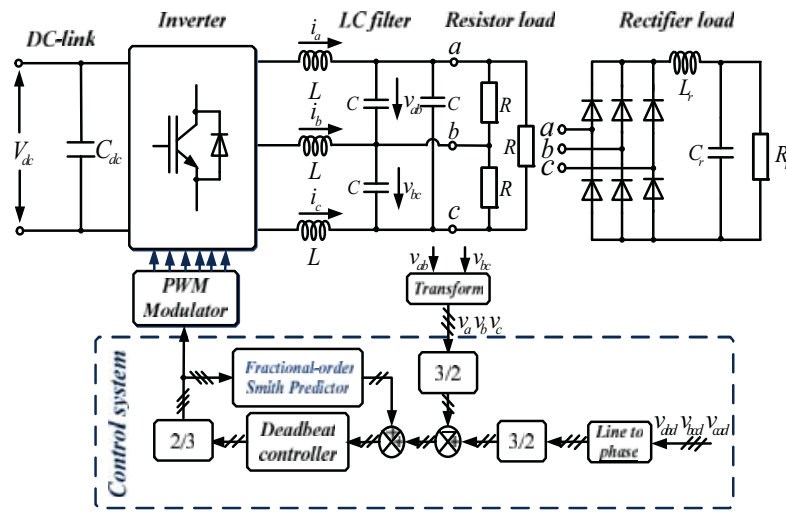

Fig. 4. Deadbeat control for three-phase PWM inverter.

$v_{A B}=V_{d c} u_{a b}, v_{B C}=V_{d c} u_{b c}$, and $v_{C A}=V_{d c} u_{c a}$ are the PWMmodulated voltages; $u_{a b}, u_{b c}$, and $u_{c a}$ are the normalized control output of the system; $V_{d c}$ is the dc-bus voltage; and $L, C$, and $R$ are the values of the inductance, capacitor, and resistance load, respectively.

Through $3 / 2$ transformation from $(a, b, c)$ to $(\alpha, \beta)$ coordinate system, (5) can be decoupled into two identical independent single-phase systems as follows:

$$
\left[\begin{array}{l}
\dot{x}_{1} \\
\dot{x}_{2}
\end{array}\right]=\left[\begin{array}{cc}
\frac{-1}{R C} & \frac{1}{3 C} \\
\frac{-1}{L} & 0
\end{array}\right]\left[\begin{array}{l}
x_{1} \\
x_{2}
\end{array}\right]+\left[\begin{array}{c}
0 \\
\frac{V_{d c}}{L}
\end{array}\right] u
$$

where $x_{1}=v_{\alpha}$ or $v_{\beta}, x_{2}=i_{\alpha}$ or $i_{\beta}, u=u_{\alpha}$ or $u_{\beta}$.

The sampled-data form of (6) can be derived:

$$
\left[\begin{array}{c}
x_{1}(k+1) \\
x_{2}(k+1)
\end{array}\right]=\left[\begin{array}{ll}
\varphi_{11} & \varphi_{12} \\
\varphi_{21} & \varphi_{22}
\end{array}\right]\left[\begin{array}{l}
x_{1}(k) \\
x_{2}(k)
\end{array}\right]+\left[\begin{array}{l}
g_{1} \\
g_{2}
\end{array}\right] u(k)
$$

where $g_{1}=\frac{V_{d c} T_{s}^{2}}{6 L C}, g_{2}=\frac{V_{d c} T_{s}}{L} \varphi_{11}=1-\frac{T_{s}}{R C}+\frac{T_{s}^{2}}{2 R^{2} C^{2}}-\frac{T_{s}^{2}}{6 L C}$

$$
\varphi_{12}=\frac{T_{s}}{3 C}-\frac{T_{s}^{2}}{6 R C^{2}}, \varphi_{21}=-\frac{T_{s}}{L}+\frac{T_{s}^{2}}{2 R L C} \text {, and } \varphi_{22}=1-\frac{T_{s}^{2}}{6 L C} \text {. }
$$

The objective is to force $v_{a b}, v_{b c}$, and $v_{c a}$ to exactly track their reference $v_{a b d}, v_{b c d}$ and $v_{c a d}$ in the presence of uncertain time delay with zero error at the sampling points.

\section{B. Design of Fractional-Order Smith Predictor Based Deadbeat Controller}

According to the theory in Section II, the digital controller for three-phase PWM inverter is comprised of two parts: a conventional deadbeat controller and a plug-in fractionalorder Smith Predictor.

1) Conventional deadbeat controller:

For the inverter system (7), the deadbeat controller is designed as follows:

$$
\begin{aligned}
u(k)= & -\frac{\varphi_{11}+\varphi_{22}}{g_{1}} x_{1}(k)-\frac{\varphi_{12} \varphi_{21}-\varphi_{22} \varphi_{11}}{g_{1}} x_{1}(k-1) \\
& -\frac{\varphi_{12} g_{2}-\varphi_{22} g_{1}}{g_{1}} u(k-1)+\frac{1}{g_{1}} v_{\text {ref }}(k)
\end{aligned}
$$

2) Fractional-order time delay compensation:

In practical applications, the performance of conventional deadbeat controller confronts severe performance 
degradation problem in the presence of model uncertainties, especially the uncertainty time-delay. Moreover, for keeping a balance between simplify and approximation accuracy, the degree $p$ of the fractional-order Smith Predictor is usually chosen as 2. Therefore, a fractional-order Smith Predictor

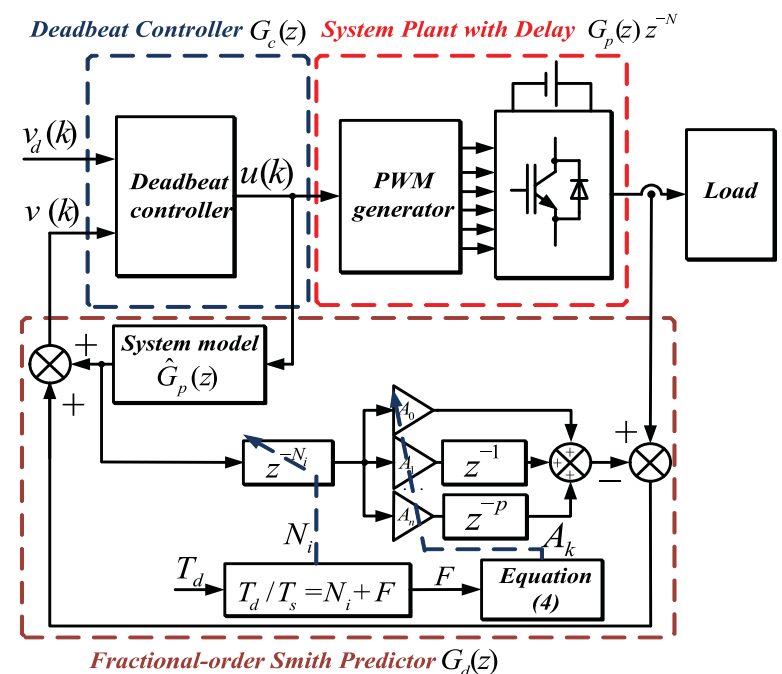

Fig. 5. Proposed fractional-order Smith Predictor based deadbeat control for three-phase PWM inverter.

can be plugged into the conventional inverter to overcome the time-delay problem as follows:

$G_{d}(z)=\hat{G}_{p}(z)\left[1+\frac{(F-1)(F-2)}{2}-F(F-2) z^{-1}+\frac{F(F-1)}{2} z^{-2}\right]$,

(9)

where $\hat{G}_{p}(z)$ is the estimation system plant.

In order to more clearly show the proposed universal delay compensation based deadbeat current control approach, implementation of the proposed scheme are illustrated in Fig. 5.

3) Simulation results:

The robustness performance of the proposed composite control solution is tested on the inverter system in the presence of various time delay cases. Nominal values of the inverter circuit parameters are given in Table 1.

Table 1 SYSTEM PARAMETERS

\begin{tabular}{l|l}
\hline Inverter & Reference \\
\hline$V_{d c}=400 \mathrm{~V}$ & $v_{a b d}=270 \sin (100 \pi t) \mathrm{V}$ \\
$L=5 \mathrm{mH}$ & $v_{b c d}=270 \sin (100 \pi t-2 \pi / 3) \mathrm{V}$ \\
$C=100 \mathrm{uF}$ & $v_{c a d}=270 \sin (100 \pi t+2 \pi / 3) \mathrm{V}$ \\
$R=100 \Omega$ & Switching frequency \\
\hline Rectifier load & $f_{s}=10 \mathrm{kHz}$ \\
\hline$L_{r}=5 \mathrm{mH}$ & $T_{s}=1 e-4 \mathrm{~s}$ \\
$C_{r}=1100 \mathrm{uF}$ & \\
$R_{r}=60 \Omega$ & \\
\end{tabular}

Fig. 6 (a) shows the steady-state response of the output voltage $v_{a b}$ and load current $i_{a}$ of the only deadbeatcontrolled three-phase inverter under three-phase rectifier load. In Fig. 6(b), the harmonic spectrum is analyzed. It can be shown that the conventional deadbeat controller works well when there are no model uncertainties. Figs. 6(c) and

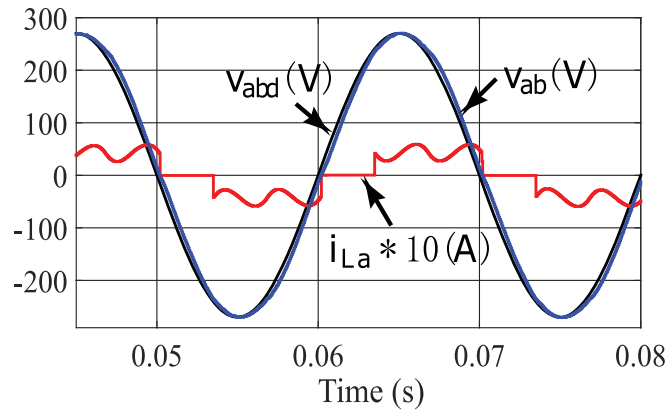

(a)

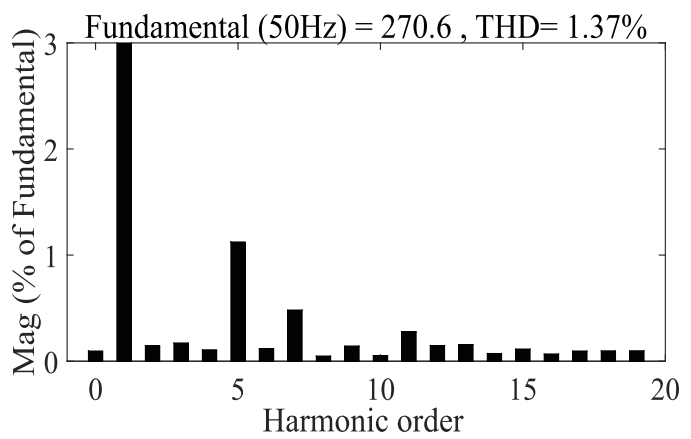

(b)

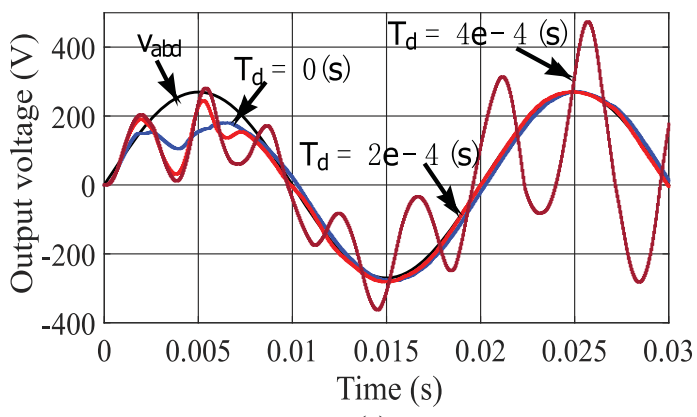

(c)

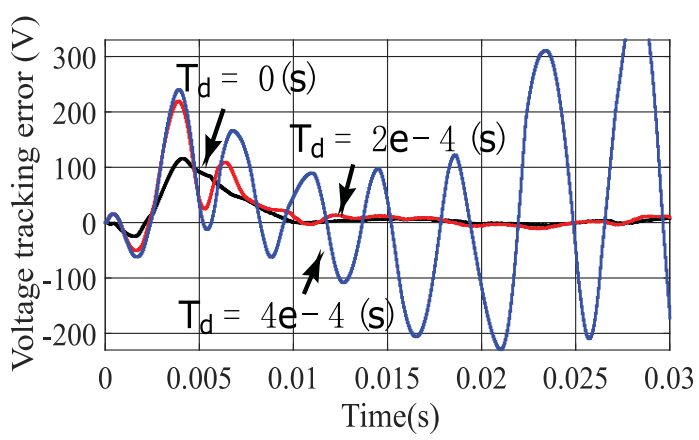

(d)

Fig. 6. Steady-state response with only deadbeat control under rectifier load. (a) Output voltage and load current. (b) Harmonic spectrum. (c) Output voltage under different time delay values. (d) Voltage tracking error under different delay values.

6(d) illustrate the responses of the output voltage, inductor current and voltage tracking error in the presence of different time delay values. Three different time delay cases are considered here (e.g. $T_{d}=0, T_{d}=2 e-4 s$, and $\left.T_{d}=4 e-4 s\right)$. It can be observed that the tracking performance degrades dramatically with the increase of time delay value. Long time delay (e.g. $T_{d}=4 e-4 s$ ) will lead to system instability when the time delay compensation approach is not employed. 


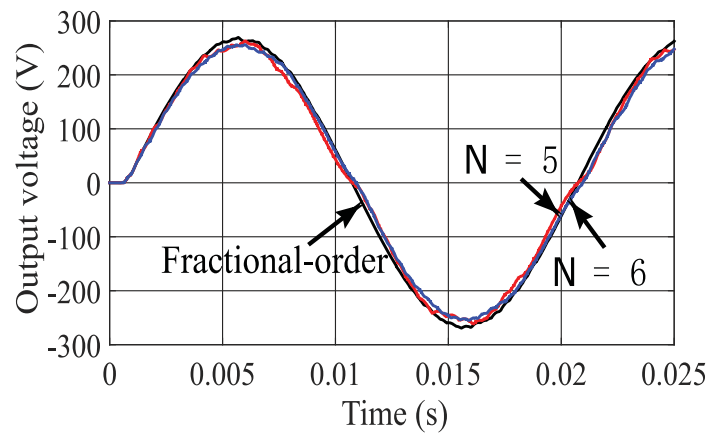

(a)

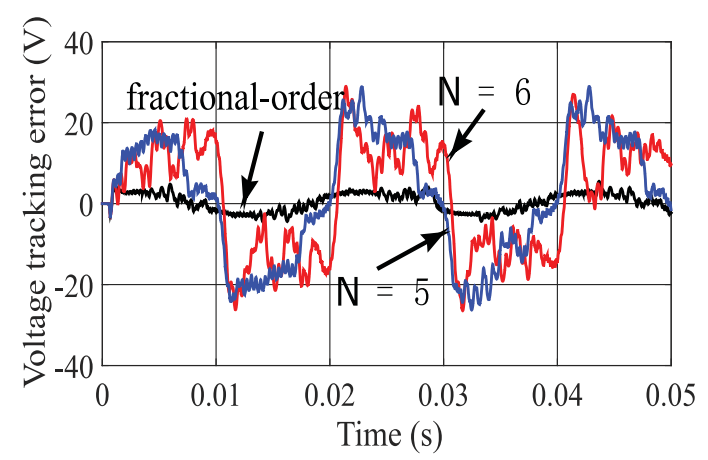

(b)

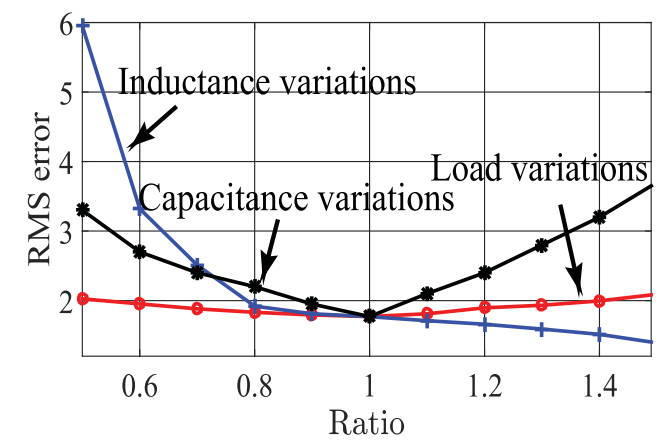

(c)

Fig. 7. Performance with time delay compensation. (a) Comparisons of voltage tracking performance with delay compensation. (b) Voltage tracking error. (c) Performance validations in the presence of parameter uncertainties with time delay.

Fig. 7 gives the responses of the deadbeat control system with conventional and improved fractional-order Smith Predictor. In this case, the time delay value here is set as $T_{d}=5.6 e-4 s$. Delay unit number $N$ is chosen as 5.6, 5 and 6 , respectively, where 5 and 6 are the two nearest integer numbers to the fractional number 5.6. It can be seen that, with the delay compensation, the system tracking performance is significantly improved than that in Fig. 6(c). Moreover, the proposed fractional-order time delay compensation achieves much better tracking accuracy than the conventional approaches, as shown in Fig. 7(b). Performance validations with up to $\pm 50 \%$ of parameter uncertainties are also investigated in this case. It can be observed that the load resistance uncertainties have only small effects on the tracking accuracy. However, when considering the effects of inductance $\mathrm{L}$ and capacitor $\mathrm{C}$, there are large impacts on the predictive accuracy of the load current. The root mean square (RMS) errors of proposed approach with delay compensation are almost at the same level with no time delay case, as shown in Fig. 7(c). In practical inverter system, it is impossible for the system parameters to have such a large range of uncertainties as studied in this case. But even so, measures to minimize the performance degradation and simplify the implementation should be investigated. In order to minimize the effects of parameter uncertainties, it is better to neglect the resistance uncertainties and select an overestimated inductance value in implementation.

\section{CONCLUSION}

In this paper, a fractional-order Smith Predictor based universal delay compensation approach is developed for voltage control of a three-phase inverter system to provide a general time delay compensation design. With the proposed unified solution, arbitrary time delay effects can be accurately approximated and compensated in the deadbeat framework. The full potential of deadbeat schemes in control accuracy and implementation simplicity can be realized, which will effectively promote widespread applications of the deadbeat schemes in practical systems. A series of case studies are carried out to demonstrate the feasibility of the proposed current control controller in a three-phase inverter system. They have shown high tracking accuracy, good transmit response, and robust time delay adaptation performance. The proposed simple but effective fractional-order time delay compensation solution can be applied without major changes to other types of control strategies.

\section{REFERENCES}

[1] M. P. Kazmierkowski, and L. Malesani, "Current control techniques for three-phase voltage-source PWM converters: A survey," IEEE Trans. Ind. Electron., vol. 45,no. 5, pp. 691-703, Oct., 1998.

[2] O. Kukrer and H. Komurcugil, "Deadbeat control method for singlephase UPS inverters with compensation of computational delay," Proc. IEEE Elect. Power Applicat., vol. 146, no. 1, pp. 123C128, Jan. 1999.

[3] P. Mattavelli, "An improved deadbeat control for UPS using disturbance observers," IEEE Trans. Ind. Electron., vol. 52, no. 1, pp. 206-212, Feb., 2005.

[4] Y. A. R. I. Mohamed, and E. F. E. Saadany, "An improved deadbeat current control scheme with a novel adaptive self-tuning load model for a three-phase PWM voltage-source inverter," IEEE Trans. Ind. Electron., vol. 54, no. 2, pp.747-759, Apr., 2007.

[5] S. Yang, Q. Lei, F. Z. Peng, and Z. Qian, "A robust control scheme for grid-connected voltage-source inverters," IEEE Trans. Ind. Electron., vol. 58, no. 1, pp. 202-212, Jan., 2011.

[6] J. C. Moreno, J. M. E. Huerta, R. G. Gil, and S. A. Gonzalez, "A robust predictive current control for three-phase grid-connected inverters," IEEE Trans. Ind. Electron., vol. 56, no. 6, pp. 1993-2004, Mar., 2009.

[7] X. Xing, Z. Zhang, C. Zhang, J. He, and A. Chen, "Space Vector Modulation for Circulating Current Suppression Using Deadbeat Control Strategy in Parallel Three-Level Neutral-Clamped Inverters," IEEE Trans. Ind. Electron., vol. 64, no. 2, pp. 977-987, Dec., 2017.

[8] Y. He, H. S. H. Chung, C. N. M. Ho, and W. Wu, "Use of boundary control with second-order switching surface to reduce the system order for deadbeat controller in grid-connected inverter," IEEE Trans. Power Electron., vol. 31, no. 3, pp. 2638-2653, Mar., 2016.

[9] J. P. Richard, "Time-delay systems: an overview of some recent advances and open problems," Automatica, vol. 39, no. 10, pp. 16671694, Oct., 2003.

[10] [10] P. Cortes, J. Rodriguez, C. Silva, and A. Flores, "Delay compensation in model predictive current control of a three-phase inverter," IEEE Trans. Ind. Electron., vol. 59, no. 2, pp. 1323-1325, Feb., 2012. 
[11] S. Majhi, and D. P. Atherton, "Obtaining controller parameters for a new Smith predictor using autotuning," Automatica, vol. 36, no. 11, pp. 1651-1658, Nov., 2000.

[12] C. L. Lai, and P. L. Hsu, "Design the remote control system with the time-delay estimator and the adaptive smith predictor," IEEE Trans. Ind. Inf., vol. 6, no. 1, pp. 73-80, Feb., 2010.

[13] J. Rodriguez, and P. Cortes, "Predictive control of power converters and electrical drives," Wiley, Hoboken, USA, 2012.

[14] B. Zhang, D. Wang, K. Zhou, and Y. Wang, "Linear phase lead compensation repetitive control of a CVCF PWM inverter," IEEE Trans. Ind. Electron., vol. 55, no. 4, pp. 1595-1602, Apr., 2008.

[15] K. Zhou, D. Wang, Y. Yang, and F. Blaabjerg, "Periodic control of power electronic converters," IET Press, London, UK, 2016.

[16] Y. Yang, K. Zhou, M. Cheng, and B. Zhang, "Phase compensation multiresonant control of CVCF PWM converters," IEEE Trans. Power Electron., vol. 28, no. 8, pp. 3923-3930, May, 2013.

[17] H. A. Young, M. A. Perez, and J. Rodriguez, "Analysis of finitecontrolset model predictive current control with model parameter mismatch in a three-phase inverter," IEEE Trans. Ind. Electron., vol. 63, no. 5, pp. 3100-3107, May, 2016.

[18] S. Vazquez, J. Rodriguez, M. Rivera, L. G. Franquelo, and M. Norambuena, "Model predictive control for power converters and drives: Advances and trends," IEEE Trans. Ind. Electron., vol. 64, no. 2, pp. 935-947, Feb., 2017.

[19] F. D. Bosio, L. A. D. S. Ribeiro, F. D. Freijedo, M. Pastorelli, and J. M. Guerrero, " Discrete-time domain modeling of voltage source inverters in standalone applications: enhancement of regulators performance by means of Smith Predictor," IEEE Trans. Power Electron., vol. 32, no. 10, pp. 8100-8114, Oct., 2017.

[20] C. L. Lai, and P. L. Hsu, "Design the remote control system with the time-delay estimator and the adaptive smith predictor," IEEE Trans. Ind. Inf., vol. 6, no. 1, pp. 73-80, Feb., 2010.

[21] M. R. Stojic, F. S. Matijevic, and L. S. Draganovic, "A robust Smith predictor modified by internal models for integrating process with dead time," IEEE Trans. Autom. Control, vol. 46, no. 8, pp. 12931298, Aug., 2001. 